\title{
SYNTHESIS OF CHITOSAN OLIGOMERS COMPOSITE SYSTEMS AND STUDY OF THEIR ACTIVITY AGAINST BIPOLARIS ORYZAE
}

\section{CASSYO ARAUJO-RUFINO ${ }^{1}$, JUCILAYNE FERNANDES-VIEIRA ${ }^{1}$, PABLO MARTÍN-RAMOS ${ }^{2,3}$, IOSODY SILVA-CASTRO ${ }^{3}$, MARCIABELA FERNANDES-CÔRREA ${ }^{3}$, PETRUTA MIHAELA MATEI $^{4}$, MERCEDES SÁNCHEZ-BÁSCONES ${ }^{4}$, M. CARMEN RAMOS-SÁNCHEZ ${ }^{5}$ and JESÚS MARTÍN-GIL ${ }^{3}$}

${ }^{1}$ Seed Science and Technology Laboratory

Federal University of Pelotas

96001-970 Pelotas, RS

Brazil

${ }^{2}$ Department of Agricultural and Environmental Sciences

Higher Polytechnic School of Huesca

University of Zaragoza

Carretera de Cuarte s/n

22071 Huesca

Spain

e-mail:pmr@unizar.es

${ }^{3}$ Department of Agricultural and Forestry Engineering

ETSIIAA, University of Valladolid

Avenida de Madrid 44

34004 Palencia

Spain

Keywords and phrases: Bipolaris oryzae, chitosan, fungicide, propolis, silver nanoparticles. Received November 16, 2015 
${ }^{4}$ Department of Agriculture and Forestry Science

ETSIIAA, Universiity of Valladolid

Avenida de Madrid 57

34004 Palencia

Spain

${ }^{5}$ Río Hortega University Hospital

Calle Dulzaina 2

47012 Valladolid

Spain

\begin{abstract}
The synthesis of composite systems of oligomeric chitosan with propolis extract which allow the incorporation of a third component (silver nanoparticles) is reported, together with their application in aqueous solutions with a view to the formation of adhesive substances or nanofilms for the protection of agricultural crops. The antimicrobial properties resulting from the association of the two biological products or from the incorporation of silver nanoparticles have been studied in vitro for rice seeds inoculated with harmful Bipolaris oryzae fungus, proposing the field application of the novel composites as biofungicides to control the brown spot disease. Several synthesis-structure-activity models have been proposed to justify the synergies and to design new polymeric materials which may represent an eco-friendly alternative to traditional chemical fungicides due to their solubility, safety and effectiveness.
\end{abstract}

\title{
1. Introduction
}

Rice (Oryza sativa L.) is a major cereal consumed by the world population, representing about $30 \%$ of worldwide grain production [1]. Bipolaris oryzae is the causal agent of rice brown spot disease and is responsible for significant economic losses, so brown spot can be deemed as one of the most important crop diseases in the world. Bipolaris oryzae is classified in the subdivision Deuteromycotina (imperfect fungi), class Euteromycetes, order Moniliales and family Dematiaceae.

Fungicide seed treatments are a must to protect the seeds and young seedlings from many seed and soil borne pathogens. Moreover, the conventional seed coating substances can be mixed with natural products, which have been reported to have protective effects on seeds 
[2]. Since the European Union has placed severe restrictions to the application of conventional chemical products to plants and seeds, there is a growing interest in the development of natural (or biodegradable) products that can be used as an alternative to chemical control in order to reduce the effects of brown spot disease on young plants, provided that they would not present a menace to the health of humans, animals and environment [3]. The use of biopolymers is ecologically viable and safe in general terms, and they are known to have antifungal effects, induce natural plant resistance and can also provide long term protection to the crop, so they constitute a particularly desirable approach for the biocontrol of phytopathogenic fungi [4].

The natural, biodegradable and biocompatible chitosan (glucosamine polymer with $\beta-1,4$ bonds), formed by the alkaline $\mathrm{N}$-deacetylation of chitin, is one the most promising candidates to synthesize novel low-cost, environmentally friendly hybrid materials, due to its ability to form films, transparency, nontoxicity and excellent adsorption features [5]. In agriculture, chitosan polymers have been used to promote plant tolerance to stress [6] and to activate defense responses to protect different plant species from pathogenic microorganisms [7], such as cucumber (Cucumis sativus L.), wheat (Triticum aestivum L.), peas (Pisum sativum L.), peanut (Arachis hypogaea L.) or rice (Oryza sativa L.) [8].

On the basis of its chemical properties, apart from inducing the immunologic system to promote resistance to plant pathogens, chitosan may also stimulate plant growth and yield [9]. Borges et al. [10] reported the suitability of chitosan to protect tomato plants from Fusarium oxysporum. In addition, chitosan has been found to have antimicrobial properties [11, 12], in which the size of its oligomers plays a key role [13].

On the other hand, propolis is known to have antimicrobial properties and has been extensively used in traditional medicine [14, 15]. Propolis is a natural resinous hive product collected by honeybees from various plant sources [16] which contains over 150 chemical species (such as coumarins, flavonoids, polyphenols, phenolic aldehydes, sesquiterpene 
quinines, amino acids, and steroids) [17]. Its strong antimicrobial activity may be due to its high content in phenols and flavonoids [14]. Propolis has also been found to have applications as an antioxidant and in food preservation [18].

Shabana et al. [3] studied the control of brown spot pathogen of rice (Bipolaris oryzae) using some phenolic antioxidants (salicylic acid, benzoic acid and hydroquinone). Hydroquinone has also been reported to be a promising antioxidant for managing seed borne pathogenic fungi of peanut [19]. Other research groups have conducted studies by combining chitosan with oils and/or plasticizers, so as to yield different types of composites or nanocomposites, such as gelatin chitosan-based edible films incorporated with clove essential oil, whose antimicrobial activity was tested against six selected microorganisms (namely, Pseudomonas fluorescens, Shewanella putrefaciens, Photobacterium phosphoreum, Listeria innocua, Escherichia coli and Lactobacillus acidophilus) [20]. Thobunluepop [2] studied the effects of various seed coating substances (chitosan lignosulphonate polymer and eugenol) on rice seed in comparison with chemical antifungal coatings. The seeds coated with biological materials were found to maintain higher sugar contents, which significantly enhanced seed storability (in contrast, under chemical fungicide stress, those compounds were lost, which directly affected seed vigor during storage).

Finally, the incorporation of nanosilver to the organic composites may also synergistically improve their antimicrobial effects, and the use of in situ synthesis methods allow its incorporation into the polymer matrix attaining uniform distributions and avoiding aggregation. Nevertheless, when nanoparticles (NPs) - such as nanosilver or nanozinc ones- are to be used as biocides on the surface of plants, the recommendations of the Joint FAO/WHO Expert Committee on Food Additives (JECFA) on the application of nanotechnologies in the food and agriculture sectors and their potential food safety implications should always be taken into consideration. 
This work aims to study in vitro the effect of chitosan oligomers -and its combinations with other substances such as propolis and/or silver nanoparticles- on the growth of Bipolaris oryzae fungus, so as to preserve the quality of seeds and their components. These new uses often require improved application systems for better established dosages and coverage of materials [21] and, consequently, we herein report the synthesis and studies on the potential activity, action mechanisms and in vitro assays with copolymers or composites of chitosan and propolis (which may also incorporate silver NPs), with a view to their field application as seed coating substances against rice seed borne fungi.

\section{Material and Methods}

\subsection{Reagents and characterization equipment}

Medium molar mass chitosan (CAS number 9012-76-4) was purchased from Sigma Aldrich Química SL (Madrid, Spain). Propolis came from Burgos region (Spain), in the Duero river basin, and has a polyphenols and flavonoids content of ca. 10\% w/v. Silver nitrate (CAS number 7761-88-8), malt extract agar (Reference 105398) and potato dextrose agar (Reference 110130) were supplied by Merck Millipore (Darmstadt, Germany). Potassium methoxide solution (25wt.\% in methanol, CAS number 865-33-8) and ethanol (puriss. p.a., ACS reagent, CAS number 64-17-5) were also purchased from Sigma Aldrich Química SL. The isolated (inoculum) Bipolaris oryzae mycelium was supplied by Universidad Federal de Pelotas (Brasil). IRGA 424 rice seeds were acquired in Pelotas, Rio Grande do Sul, Brazil.

An ultrasonic machine, model CSA 20-S500, 20KHz was used for the sonication of the solutions. 


\subsection{Synthesis of solutions of chitosan oligomers, chitosan oligomers/propolis and chitosan oligomers/propolis/Ag NPs}

\subsubsection{Chitosan oligomers preparation $\left(A_{50}\right)$}

Chitosan oligomers aqueous solutions were prepared from a solution of commercial medium molar mass chitosan with molar masses in the $190000-310000 \mathrm{~g} / \mathrm{mol}$ range in $\mathrm{AcOH} 2 \%$ at $\mathrm{pH}$ 4-6. The hydrolysis was performed by stirring for 12 hours followed by 3-6 sonication periods (5 minutes each), at temperatures in the 30 to $60^{\circ} \mathrm{C}$ range and with $\mathrm{H}_{2} \mathrm{O}_{2}$ concentrations ranging from 0.3 to $0.6 \mathrm{M}$, obtaining oligomers with molar masses in the 6000 to $2000 \mathrm{~g} / \mathrm{mol}$ range, respectively, in agreement with the analogous microwave-based procedure reported by Sun et al. [22]. The molar mass of the chitosan samples was determined by measuring the viscosity, in agreement with Yang et al. [23], in a solvent of $0.20 \mathrm{~mol} / \mathrm{L} \mathrm{NaCl}+0.1 \mathrm{~mol} / \mathrm{L} \mathrm{CH}_{3} \mathrm{COOH}$ at $25^{\circ} \mathrm{C}$ using an Ubbelohde capillary viscometer. Molar masses were determined using the MarkHouwink equation $[\eta]=1.81 \times 10^{-3} \mathrm{M}^{0.93}$ [24]. The solutions were then decanted to remove any water insoluble material, were allowed to rest till cloudiness was observed and were centrifuged to isolate the chitosan

oligomers. These were re-dissolved again in $\mathrm{AcOH} 0.5 \%$, obtaining the solutions for the assays.

Aqueous solutions of chitosan oligomers $0.005-0.01 \mathrm{M}$ or solutions at $1.25-2.5 \% \mathrm{w} / \mathrm{v}$ (solution $A_{50}$ ) were prepared from a solution of chitosan oligomers with $2000 \mathrm{~g} / \mathrm{mol}$ molar mass in $\mathrm{AcOH}$ at $0.5 \%$ and $\mathrm{pH} 4-6$, adjusted with some dropplets of $\mathrm{KOCH}_{3} 25 \%$ in methanol. The chitosan/AcOH/water mixture was sonicated for a minute and was subsequently stirred for 12 hours, resulting in a transparent and stable chitosan solution. The solution was stored in inert atmosphere at $4^{\circ} \mathrm{C}$ at $\mathrm{pH} 5$ till it was used. The characterization of the different products can be found in [25]. 


\subsubsection{Propolis extraction $(P)$}

The propolis solution was prepared by grinding raw propolis to fine powder and subsequent extraction of the active ingredients by maceration in a hydroalcoholic solution 7:3 (v/v) for one week at room temperature. A hydroalcoholic medium was chosen over absolute ethanol because it results in wax-free tinctures containing higher amounts of polyphenolic substances [26]. The resulting solution was then percolated ( $1 \mathrm{~L} / \mathrm{min}$ ) and filtrated with a stainless steel mesh to remove any residue, followed by concentration at a temperature below $60^{\circ} \mathrm{C}$ with ultrasound equipment to finally obtain a clarified propolis extract, and finally solutions with a propolis concentration of $100 \mathrm{mg} / \mathrm{mL}$ or $10 \% \mathrm{w} / \mathrm{v}$ were prepared (labelled as $P$ ).

\subsubsection{In situ preparation of chitosan/propolis mixtures $\left(B_{100}\right)$}

A $50 \mathrm{~mL}$ solution of $A_{50}$ (i.e., $2.5 \% \mathrm{w} / \mathrm{v}$ of chitosan in water) was mixed with $50 \mathrm{~mL}$ of a solution of $2.5 \% \mathrm{w} / \mathrm{v}$ of propolis in water/alcohol, and the resulting mixture $(100 \mathrm{~mL})$ was sonicated for 1 minute.

\subsubsection{Silver NPs preparation}

Silver nanoparticles were prepared by a sonication method, without resourcing to UV stabilization (used, e.g., by Montazer et al. [27]) as follows: an aqueous solution of $\mathrm{AgNO}_{3}(50 \mathrm{mM})$ was treated with sodium citrate $(30 \mathrm{mM})$ and the resulting solution was cooled and stirred at a temperature between 5 and $10^{\circ} \mathrm{C}$. Subsequently, it was deoxygenated with an inert gas $\left(\mathrm{N}_{2}\right)$ for over 30 minutes and the $\mathrm{pH}$ was adjusted between 7 and 8 . Polyvinylpyrrolid one was added to prevent the silver nanoparticles aggregation. A $10 \mathrm{mM}$ solution of $\mathrm{NaBH}_{4}$ (reducing agent) was then added dropwise; the first droplet made the solution turn from colourless to yellowish and successive droplets led to an intensification of the yellow colour (care had to be taken so as to avoid an excess of reducing agent, which would lead to a brownish colour). After vigorous stirring for one hour, the yellowish solution was sonicated for 3-5 minutes and then allowed to rest and stabilize for at least 24 hours in a refrigerator at $5^{\circ} \mathrm{C}$. 
The resulting sonicated solutions had silver contents ranging from $100 \mathrm{ppm}$ to 200ppm (nAg solution) and were characterized by UV-Vis absorbance at 420nm with a Shimazdu UV-2450 UV-Vis spectrophotometer. The silver nanoparticles size was studied by SEM and TEM with a FEI-Quanta 200FEG and a JEOL JEM-FS2200 HRP, respectively [25]. The solutions were stable in inert atmosphere at $4^{\circ} \mathrm{C}$.

\subsubsection{In situ preparation of chitosan/propolis/silver NPs mixtures $\left(C_{1}, C_{2}\right)$}

The chitosan oligomers/propolis solutions $\left(B_{100}\right)$ were prepared according to Subsection 2.2.3. Two different $50 \mathrm{~mL}$ solutions of silver nanoparticles, with a concentration of $10 \mathrm{ppm}$ and $20 \mathrm{ppm}$, respectively, were also prepared. The silver NPs solutions were then added to the chitosan oligomers/propolis solutions. The resulting solutions were sonicated for a minute, so as to obtain in situ the two new mixtures of chitosan/propolis/silver NPs solutions, labelled as $C_{1}$ (for $10 \mathrm{ppm} \mathrm{nAg}$ ) and $C_{2}$ (for $20 \mathrm{ppm} \mathrm{nAg}$ ).

\subsubsection{In situ preparation of chitosan/silver NPs mixtures $\left(D_{1}, D_{2}\right)$}

$50 \mathrm{~mL}$ of $A_{50}$ chitosan oligomers solution were mixed with $50 \mathrm{~mL}$ of $10 \mathrm{ppm}$ silver NPs solution, yielding $D_{1}$ solution, and another $50 \mathrm{~mL}$ of $A_{50}$ were mixed with $50 \mathrm{~mL}$ of $20 \mathrm{ppm}$ silver NPs solution, yielding $D_{2}$ solution. Both mixtures were sonicated for a minute.

\subsubsection{In situ preparation of propolis/silver mixtures $\left(F_{1}, F_{2}\right)$}

Two $50 \mathrm{~mL}$ hydroalcoholic propolis solutions $(2.5 \% \mathrm{w} / \mathrm{v})$ were mixed with $50 \mathrm{~mL}$ of $10 \mathrm{ppm}$ silver NPs solution, yielding $D_{1}$ solution, and $50 \mathrm{~mL}$ of 20ppm silver NPs solution, yielding $D_{2}$ solution, respectively. The resulting mixtures were sonicated for a minute. 


\subsection{Culture media and activity assays}

\subsubsection{Identification and cultivation of isolates}

Conidia of Bipolaris oryzae isolates were induced to germinate in Petri dishes containing agar and water, followed by incubation in a growth chamber with controlled temperature $\left(25^{\circ} \mathrm{C}\right)$ and photoperiod $(12 \mathrm{~h}$ of light/12h of darkness) for 3-4 hours. The morphology of conidia and conidiophore, spore germination, position and direction of growth of the germ tube and ontogeny of septa were checked [28]. The isolates were grown in potato dextrose agar (PDA). After 14 days, the conidial length and width and the number of septa of each isolate were assessed.

\subsubsection{Inoculum preparation}

The fungal isolates were cultured in Petri dish containing PDA culture medium and incubated for 14 days in aforementioned growth chamber. $10 \mathrm{~mL}$ of sterile distilled water were then added to each Petri dish and mass spores were homogenized with the aid of a sterilized brush. The suspension, after filtering (with a funnel with gauze filter), was collected in a test tube. Then the suspension was standardized at 2000 spores/mL by using a Neubauer chamber. Seeds inoculation was conducted by immersion in the spore suspension in the Petri dishes for 48 hours in the growth chamber.

\subsubsection{Seed health testing}

According to ISTA [29] standard Blotter and agar plate tests, seeds were incubated for a definite period under specific conditions. The fungicidal action of the products under study was tested in vitro using potato dextrose agar (PDA) as a culture medium. The nine solutions described above $\left(A_{50}, B_{100}, C_{1}, C_{2}, D_{1}, D_{2}, F_{1}, F_{2}, P\right)$ were used as treatments against Bipolaris oryzae fungus at three different concentrations $(300 \mu \mathrm{L} / \mathrm{mL}, 600 \mu \mathrm{L} / \mathrm{mL}$, and $900 \mu \mathrm{L} / \mathrm{mL})$ in PDA at $25^{\circ} \mathrm{C}$ and compared with the control $(T)$. The seeds were incubated at $20 \pm 2^{\circ} \mathrm{C}$ for seven days under a photoperiod regime of 12 hours of light/12 hours of darkness. The treatment of the seeds inoculated with Bipolaris oryzae was conducted by spraying at a dose of $1 \mathrm{~mL} / 100 \mathrm{~g}$ of seeds. 2000 inoculated rice (Oryza 
sativa cv. IRGA424) seeds were validated and distributed in 8 repetitions of 25 seeds per 10 treatments $\left(A_{50}, B_{100}, C_{1}, C_{2}, D_{1}, D_{2}, F_{1}, F_{2}, P, T\right)$. The 25 inoculated seeds of each repetition were distributed in germitest paper rolls, previously pressed to obtain $0.5 \mathrm{~cm} \times 0.5 \mathrm{~cm}$ capsules, and placed in $11.5 \mathrm{~cm} \times 11.5 \mathrm{~cm} \times 3.5 \mathrm{~cm}$ transparent plastic boxes with lids, previously disinfected with a sodium hypochlorite $1 \%$ solution. The germitest paper rolls were soaked with an amount distilled water equivalent to 2.5 times the weight of the paper used. After an incubation period of 14 days at $20-25^{\circ} \mathrm{C}$ with $12 \mathrm{~h}$ of light, the seeds were individually examined for fungal growth using a stereomicroscope and the incidence of pathogenic fungi was expressed as the percentage of infected seeds.

\subsubsection{Determination of the inhibition percentage for Bipolaris oryzae fungus}

Growth measurements of the diameter of the fungal mycelium were performed in triplicate $\left(R_{1}, R_{2}\right.$ and $\left.R_{3}\right)$ to determine the degree of sensitivity/resistance to each of the products $\left(A_{50}, B_{100}, C_{1}, C_{2}, D_{1}, D_{2}\right.$, $\left.F_{1}, F_{2}, P, T\right)$. The diameter of fungal growth was measured on a daily basis for 20 days, and the inhibition percentage (IP) was calculated taking the pure MEA culture (control) as a reference according to the following equation [30]:

$$
I P(\%)=\frac{D m t-D v a r}{D m t} \cdot 100
$$

where Dmt is the diameter of the mycelium in the control (pure MEA) and Dvar is the diameter of the mycelium of the sample mixed with one of the antimicrobial composites.

\subsubsection{Germination test}

The seeds were treated with the different combinations of active products (viz. $A_{50}, B_{100}, C_{1}, C_{2}, D_{1}, D_{2}, F_{1}, F_{2}, P$ ) and 4 repetitions with 100 seeds were carried out for each treatment (plus control), according to ISTA [29]. The results were expressed as the percentage of normal germinated seedlings. 


\subsubsection{Seedlings stem and root dimensions}

Stem and root lengths (in $\mathrm{mm}$ ) were measured -with a graduated scale in millimeters- for 10 normal seedlings randomly chosen from 4 replications of 20 seeds per treatment, which had been placed to germinate in the germitest paper rolls, according to the method described above. The arithmetic mean of the stem and root lengths were then calculated and the results were expressed in $\mathrm{cm}$ [31].

\subsubsection{Statistical analysis}

The trial was arranged in a completely randomized design with 4 repetitions, totaling 40 experimental units. The experimental unit was characterized by Petri plates and germitest paper rolls. Data were analyzed by ANOVA and by Tukey's HSD test at 5\% significance level. Statistical analyses were performed by using the statistical program SASM-Agri [32].

\section{Results and Discussion}

\subsection{Treatment of rice seeds against Bipolaris oryzae fungus}

\subsubsection{Antifungal properties}

In Table 1, it may be observed that, even at a concentration of $300 \mu \mathrm{L} / \mathrm{mL}$, a complete inhibition of Bipolaris oryzae growth $(\varnothing=0 \mathrm{~cm})$ was attained for chitosan oligomers $\left(A_{50}\right)$, chitosan/propolis $\left(B_{100}\right)$, chitosan/propolis/nAg $\left(C_{1}\right.$ and $\left.C_{2}\right)$ and chitosan/nAg $\left(D_{1}\right.$ and $\left.D_{2}\right)$ treatments. For the propolis $(P)$ and propolis/nAg $\left(F_{1}\right.$ and $\left.F_{2}\right)$ treatments, higher concentrations $(600 \mu \mathrm{L} / \mathrm{mL})$ were required so as to achieve complete inhibition of the fungus. 
Table 1. Mycelium diameter for Bipolaris oryzae fungus as a function of concentration for the different treatments: $A_{50}, B_{100}, C_{1}, C_{2}, D_{1}, D_{2}$, $F_{1}, F_{2}, P$ and the control

\begin{tabular}{cccc}
\hline & \multicolumn{3}{c}{ Concentration $(\mu \mathrm{L} / \mathrm{mL})$} \\
\cline { 2 - 4 } Treatment & 300 & 600 & 900 \\
\hline$A_{50}$ & 0 & 0 & 0 \\
$B_{100}$ & 0 & 0 & 0 \\
$C_{1}$ & 0 & 0 & 0 \\
$C_{2}$ & 0 & 0 & 0 \\
$D_{1}$ & 0 & 0 & 0 \\
$D_{2}$ & 0 & 0 & 0 \\
$F_{1}$ & 5.7 & 0 & 0 \\
$F_{2}$ & 5.7 & 0 & 0 \\
$P$ & 6.8 & 0 & 0 \\
\hline Control & 7.4 & 7.8 & 7.1 \\
\hline Coefficient of variation (\%) & 4.5 & 3.5 & 4.1 \\
\hline
\end{tabular}

Figure 1 shows an example of the radial growth of the fungus in Petri dishes, depicting the control and the results obtained at different concentrations of one of the treatments $\left(C_{2}\right)$. It can be readily observed that, whereas the fungus covers the entire Petri dish for the control (right), nomycelial growth took place for the seeds treated with the chitosan/propolis/nAg composite, regardless of the concentration. 


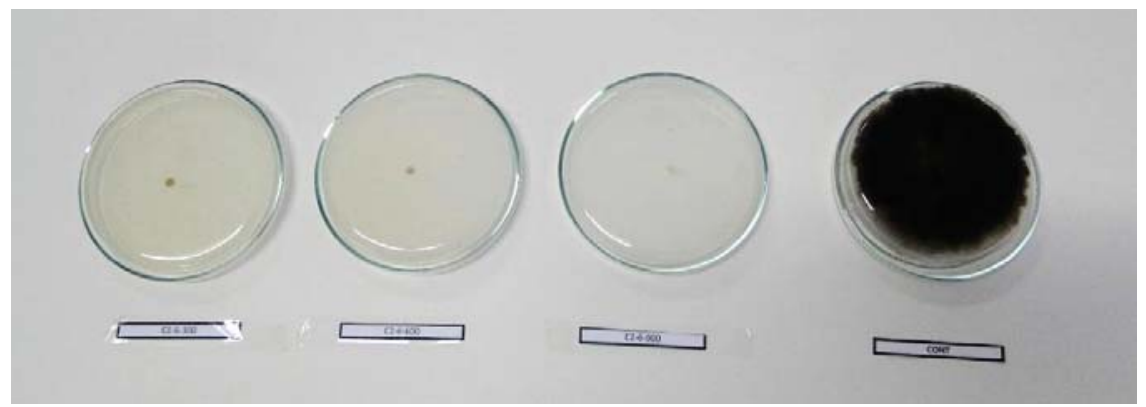

Figure 1. Growth of Bipolaris oryzae fungus in Petri dishes. From left to right: Petri dishes with no fungal growth at concentrations of 300, 600, and $900 \mu \mathrm{L} / \mathrm{mL}$ for $C_{2}$ treatment and Petri dish with complete growth of the fungus for the control.

According to Figure 2, the chitosan oligomers $\left(A_{50}\right)$, chitosan/ propolis $\left(B_{100}\right)$, chitosan/propolis/nAg $\left(C_{1}\right.$ and $\left.C_{2}\right)$ and chitosan/nAg $\left(D_{1}\right.$ and $\left.D_{2}\right)$ combinations would be particularly active, with maximum inhibition percentages (IP). The lower efficacy of propolis $(P)$ and propolis/nAg $\left(F_{1}\right.$ and $\left.F_{2}\right)$ treatments can be attributed to a decrease in solubility due to the use of hydroalcoholic solutions for propolis conveying. This suggests that subsequent studies should focus on improving the solubility, for example, by replacing the current intermolecular bonding system (cross-linkage) with the formation of graft copolymers (between chitosan and propolis oils), in line with the studies of Tiwary et al. [33] and Gómez-Estaca et al. [20], as it will be further discussed in Subsection 3.2. 
(a)

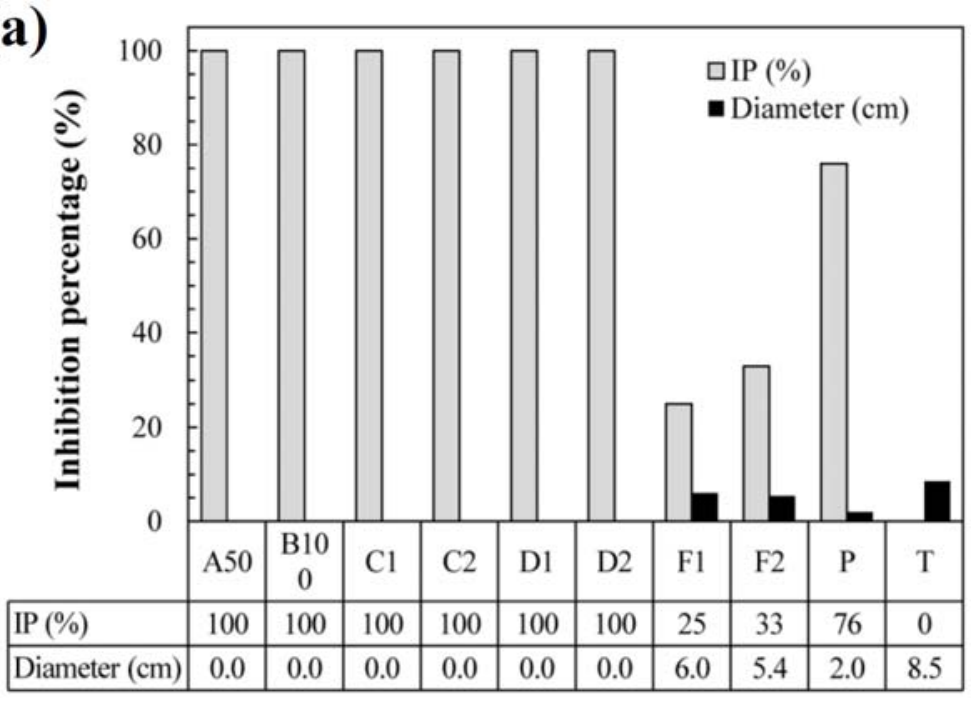

(b)

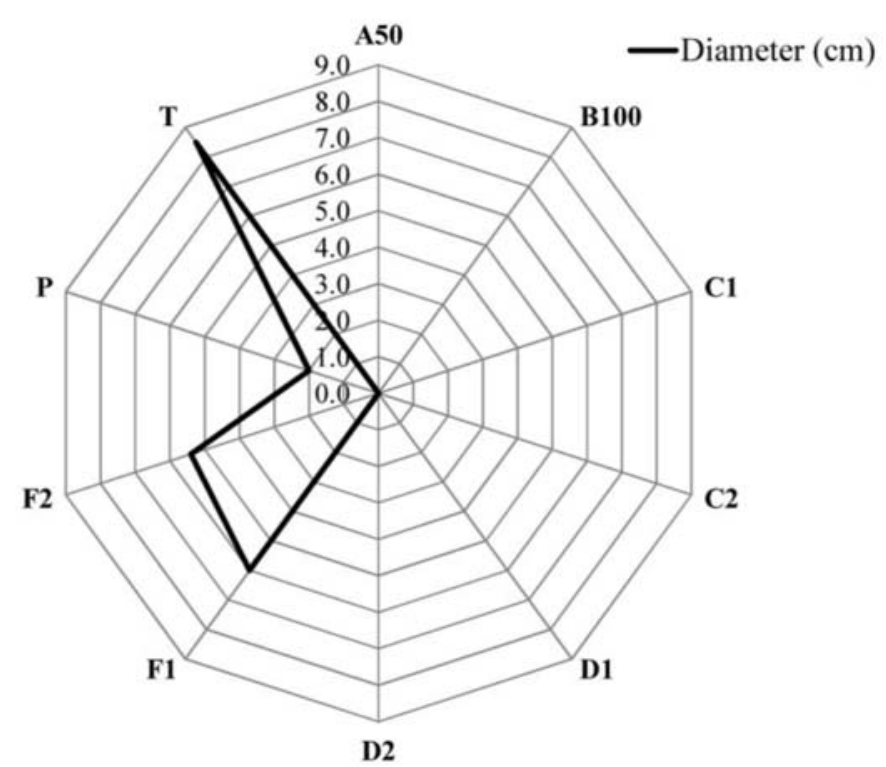

Figure 2. (a) Inhibition percentage (IP) and (b) growth diameter of Bipolaris oryzae fungus for the treatments with chitosan oligomers $\left(A_{50}\right)$, chitosan/propolis $\left(B_{100}\right)$, chitosan/propolis/nAg $\left(C_{1}\right.$ and $\left.C_{2}\right)$, chitosan/nAg $\left(D_{1}\right.$ and $\left.D_{2}\right)$, propolis/nAg $\left(F_{1}\right.$ and $\left.F_{2}\right)$, propolis $(P)$ and control $(T)$. 
Several possible mechanisms have been proposed to explain the antibacterial properties of chitosan: it is known that positively charged amine groups are capable of interacting with the negatively charged bacterial cell membrane and, in addition, chitosan may also bind to DNA, leading to inhibition of mRNA and proteins synthesis [34]. The increase in the antimicrobial activity of the new composites with chitosan oligomers may be due to chemical interaction of the amine and hydroxyl groups with nanosilver. It has also been demonstrated that polymeric chitosan and chitosan oligomers induce phytoalexins that help limit the spread of pathogens [35]. Chemical synthesis of different sizes of chitosan oligomers with specific biological activity has been described by Kuyama et al. [36].

For mixtures of pure propolis extract $(P)$ or the propolis/silver NPs combinations $\left(F_{1}\right.$ and $\left.F_{2}\right)$, their efficiency must be referred to the presence of phenolic groups [37] and to enzyme inhibition by nonspecific interactions with proteins [38].

Silver nanoparticles have been deemed as one of the most promising antimicrobial species from a nanotechnology based approach, since their activity is very broad and is well above that of raw silver. For example, silver ions can bind to negatively charged bacterial peptidoglycan walls and can diffuse into bacterial cells and bind to DNA bases, leading to bacterial death and/or inhibiting the replication and transcription processes and preventing further bacterial production [39]. Moreover, the generation of reactive oxygen species, which leads to nanotoxicity processes, is also a well-established antimicrobial mechanism. The main disadvantages that would limit the use of nanosilver are its ease of aggregation and the uncontrolled release of silver ions and their cytotoxicity potential [40], which are not an issue in this case.

\subsubsection{Phytotoxicity tests}

The phytotoxicity of the different combinations (vs. the control) was assessed through the results of the germination tests and the measurement of stem and root lengths, summarized in Table 2. From these results, it can be inferred that -for the germination of rice seeds- 
chitosan/silver NPs $\left(D_{2}\right)$ and propolis $(P)$ were the two treatments that led to the highest germination indices ( $91 \%$ and $90 \%$, respectively), significantly different from that obtained for the control (85\%). For the rest of the treatments, it can be concluded that the antifungal properties are not accompanied by the stimulating properties for germination or for seedling growth that were initially expected.

Table 2. Results from the germination tests and stem and root lengths for seeds treated with different combinations of chitosan/propolis/silver NPs: $A_{50}, B_{100}, C_{1}, C_{2}, D_{1}, D_{2}, F_{1}, F_{2}, P$ and control

\begin{tabular}{cccc}
\hline Treatment & $\begin{array}{c}\text { Germination } \\
\text { test (\%) }\end{array}$ & $\begin{array}{c}\text { Stem length } \\
(\mathrm{cm})\end{array}$ & $\begin{array}{c}\text { Root length } \\
(\mathrm{cm})\end{array}$ \\
\hline$A_{50}$ & 85 & 3.36 & 8.33 \\
$B_{100}$ & 65 & 2.81 & 8.75 \\
$C_{1}$ & 85 & 2.87 & 10.15 \\
$C_{2}$ & 85 & 2.71 & 9.57 \\
$D_{1}$ & 82 & 3.76 & 8.89 \\
$D_{2}$ & 91 & 3.92 & 9.23 \\
$F_{1}$ & 60 & 2.86 & 9.87 \\
$F_{2}$ & 80 & 2.74 & 9.31 \\
$P$ & 90 & 4.18 & 10.54 \\
\hline Control & 85 & 4.14 & 10.45 \\
\hline Coefficient of variation (\%) & 2.5 & 3.6 & 3.1 \\
\hline
\end{tabular}

\subsection{Synthesis-structure-activity relationships}

\subsubsection{Chitosan oligosaccharides-based nanocomposites}

Although -as noted above- chitosan is a source of potential bioactive material, it has several drawbacks for its direct utilization in biological applications, including its poor solubility under physiological conditions and a high viscosity in dilute acidic solutions [41]. In contrast, the hydrolyzed products of chitosan, such as its oligomers, have better 
solubility and lower viscosity, because of their shorter chain lengths and the free amino groups in the D-glucosamine units. A weight-average molecular weight (MW) ranging from $10,000 \mathrm{Da}$ to about $100,000 \mathrm{Da}$ is considered for low MW chitosan, while the MW of oligochitosan is generally lower than 10,000 $\mathrm{Da}$ [42]. The activity of chitosan is closely correlated with its structure and physicochemical properties, such as its degree of deacetylation, degree of polymerization and cationic nature, which are improved in the oligomers [43]. Sonication has become an alternative for degrading chitosan into low-molecular-weight chitosan, chitosan oligomers and glucosamine. The degree of deacetylation tends to decrease due to the fact that the amino groups on the C-2 of chitosan facilitate the site-specific fragmentation of the glycosidic linkage during $\beta$-cleavage after the sonication treatment [44].

\subsubsection{Grafting and crosslinking mechanisms for the formation of chitosan based nanocomposites}

Different chitosan products have different structures and physicochemical properties, which may result in novel bioactivities or novel findings in known bioactive compounds [45]. Chemical modification of chitosan/chitosan oligomers can be attained by $\mathrm{N}$-substitution, by O-substitution, by N,O-substitution and also via chitosan association with small molecules or macromolecules. In this regard, chitosan can be deemed as a promising material, since it contains three types of reactive functional groups: an amino/acetamide group as well as both primary and secondary hydroxyl groups at the C-2, C-3 and C- 6 positions. The amino contents are the main reason for the differences between their structures and physicochemical properties, which are correlated with their chelation, flocculation and biological functions [43] (Figure 3). 


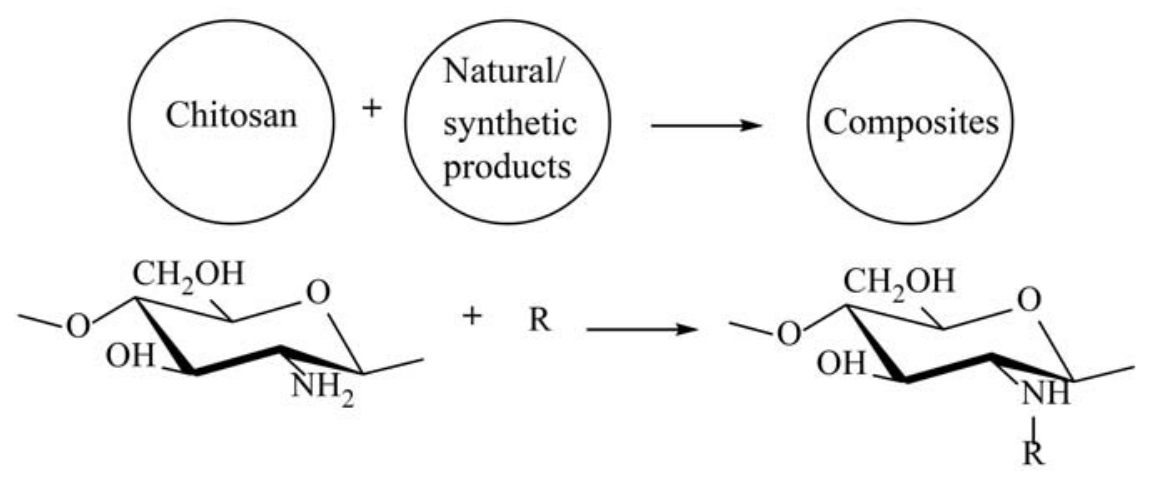

Figure 3. Composite formation from the D-glucosamine structure by addition of a functional group.

Chitosan carries free amine functionalities on the deacetylated units and hydroxyl groups on the acetylated as well as deacetylated units. The modification of chitosan by introduction of small functional groups such as alkyl or carboxymethyl groups can increase its solubility at neutral and alkaline $\mathrm{pH}$ without affecting its cationic character. Thus, chitosan can be grafted with other molecules through covalent binding (Figure 4(a)). The amino groups can be used for acetylation, quaternization, reactions with aldehydes and ketones, chelation of metals, etc., while the hydroxyl groups can lend to o-acetylation, H-bonding with polar atoms, etc. [33].

Chitosan is a cationic polysaccharide due to the presence of the amino group, which conferms more reactivity and the ability to bind functional groups that appear in natural or synthetic extracts, resulting in the formation of novel compounds. 
(a)

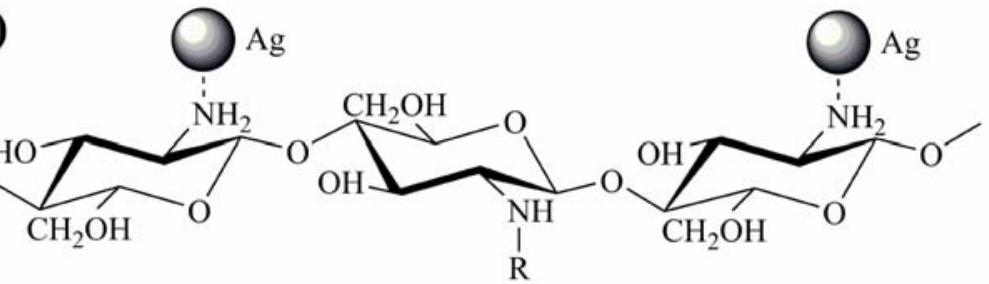

(b)

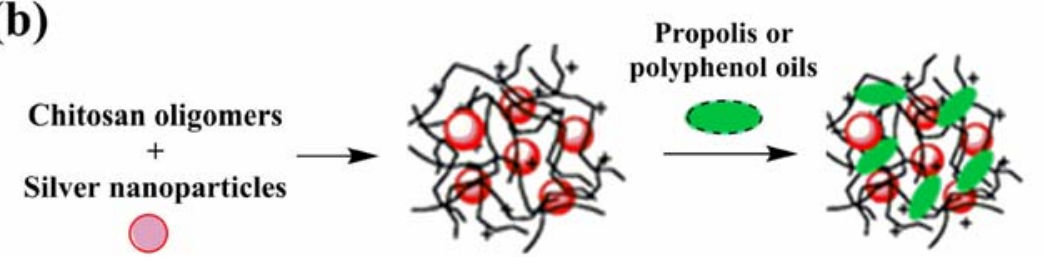

Figure 4. Mechanisms for the formation of chitosan based nanocomposites: (a) composite formation from chitosan oligomers grafted to propolis or polyphenol oils (R) and silver nanoparticles (Ag); (b) composite formation by electrostatic interactions and intermolecular hydrogen bonding between chitosan, propolis and silver nanoparticles.

Chitosan may also be cross-linked with natural extracts by electrostatic interactions and intermolecular hydrogen bonding (Figure 4(b)). In recent years, the unparalleled and functional properties of essential oils have been extensively reported, but the sensitivity of essential oils to environmental factors and their poor aqueous solubility have limited their applications in industries. Carum copticum essential oil was combined with chitosan nanoparticles by an emulsion ionic gelation, using pantasodium tripolyphosphate and sodium hexametaphosphte as cross-linkers. The biological properties of Carum copticum essential oil, before and after the encapsulation process, were evaluated by FTIR and thermal analysis. The results indicated that the essential oil had been encapsulated into the chitosan nanoparticles without any chemical reaction. The structure and function of oil were not changed in this process, suggesting maintenance of its antibacterial and antioxidant properties [46]. Thyme oil has also been mixed with chitosan in order to make biofilms, for potential applications of wound dressing. 
The antimicrobial and the antioxidant activities of the films were also investigated. The results revealed that thyme oil had a good potential to be incorporated into chitosan to make antibacterial and permeable films for wound healing applications. The FTIR spectra of chitosan films incorporated with different amounts of thyme oil showed the same pattern on their informative peaks as the control chitosan films, thus indicating that there was no interaction between active groups of thyme oil with functional groups of chitosan [47]. Matei et al. [25] conducted the synthesis of chitosan oligomers/propolis/silver nanoparticles composite systems and studied their activity against a xylophagous fungus (Diplodia seriata). In that case, and also for the treatments assayed in the study reported herein, the ATR-FTIR vibrational characterization suggested the existence of hydrogen bonding between chitosan and propolis.

Our results and those related in the literature suggest that chitosanpropolis graft copolymers would feature an enhanced stability and solubility in comparison to the copolymers based in crosslinking or intermolecular hydrogen bonds. Therefore, future lines of research should place emphasis on the development of the former composites.

\section{Conclusion}

The effect of chitosan oligomers and their combination with propolis and/or silver nanoparticles was studied in vitro against a seed borne pathogenic fungus: Bipolaris oryzae. The results were conclusive in showing that chitosan oligomers and the composites of chitosan with

propolis extract and silver nanoparticles led to a remarkable increase of the plant resistance against microbial pathogens. The importance of this finding lies in the fact that it is a step towards the goal of decreasing the use of chemical fungicides in plant pathology. From germination tests, it could be concluded that the best results (in comparison to the other treatments) corresponded to the treatment with chitosan/silver NPs composite, with a germination rate of $91 \%$. The novel polymeric materials 
can thus be deemed as very promising for the control of the brown spot disease of rice and pave the way towards eco-friendly alternatives to chemical fungicides. Further research aimed at the preparation of chitosan/ propolis graft copolymers and to assess the shelf-life of the different seed coating substances during storage is under way.

\section{References}

[1] B. K. Yadav and V. K. Jindal, Changes in head rice yield and whiteness during milling of rough rice (Oryza sativa L.), J. Food Eng. 86 (2008), 113-121.

[2] P. Thobunluepop, Characterization of a botanical fungicide from Thai origin and its efficiency in rice production, in: Crop Sciences, Georg-August University of Göttingen, Cuvillier Verlag, Göttingen, Germany, 2008.

[3] Y. M. Shabana, G. M. Abdel-Fattah, A. E. Ismail and Y. M. Rashad, Control of brown spot pathogen of rice (Bipolaris oryzae) using some phenolic antioxidants, Braz. J. Microbiol. 39 (2008), 438-444.

[4] C. Tan, B. Feng, X. Zhang, W. Xia and S. Xia, Biopolymer-coated liposomes by electrostatic adsorption of chitosan (chitosomes) as novel delivery systems for carotenoids, Food Hydrocolloids 52 (2016), 774-784.

[5] M. N. V. Ravi Kumar, A review of chitin and chitosan applications, React. Funct. Polym. 46 (2000), 1-27.

[6] S. Lee, H. Choi, S. Suh, I.-S. Doo, K.-Y. Oh, E. Jeong Choi, A. T. Schroeder Taylor, P. S. Low and Y. Lee, Oligogalacturonic acid and chitosan reduce stomatal aperture by inducing the evolution of reactive oxygen species from guard cells of tomato and commelina communis, Plant Physiol. 121 (1999), 147-152.

[7] N. Benhamou, Elicitor-induced plant defence pathways, Trends Plant Sci. 1 (1996), 233-240.

[8] W. Lin, X. Hu, W. Zhang, W. John Rogers and W. Cai, Hydrogen peroxide mediates defence responses induced by chitosans of different molecular weights in rice, J. Plant Physiol. 162 (2005), 937-944.

[9] S. Boonlertnirun, C. Boonraung and R. Suvanasara, Application of chitosan in rice production, Journal of Metals, Materials and Minerals 18 (2008), 47-52.

[10] A. A. Borges, A. Borges, G. Cabrera, A. Falcón, A. Gutiérrez, D. Paz-Lago and M. A. Ramírez, Tomato- Fusarium oxysporum interactions: II, Chitosan and MSB induced resistance against fol in young tomato plants, (2000).

[11] J. Rhoades and S. Roller, Antimicrobial actions of degraded and native chitosan against spoilage organisms in laboratory media and foods, Appl. Environ. Microbiol. 66 (2000), 80-86. 
[12] L. Y. Chung, R. J. Schmidt, P. F. Hamlyn, B. F. Sagar, A. M. Andrew and T. D. Turner, Biocompatibility of potential wound management products: Fungal mycelia as a source of chitin/chitosan and their effect on the proliferation of human F1000 fibroblasts in culture, Journal of Biomedical Materials Research 28 (1994), 463-469.

[13] L. A. Hadwiger, T. Ogawa and H. Kuyama, Chitosan polymer sizes effective in inducing phytoalexin accumulation and fungal suppression are verified with synthesized oligomers, Molecular Plant-microbe Interactions: MPMI 7 (1994), 531-533.

[14] N. Nedji and W. Loucif-Ayad, Antimicrobial activity of Algerian propolis in food borne pathogens and its quantitative chemical composition, Asian Pacific Journal of Tropical Disease 4 (2014), 433-437.

[15] V. Bankova, Recent trends and important developments in propolis research, Evidence-Based Complementary and Alternative Medicine 2 (2005), 29-32.

[16] E. Ghisalberti, Propolis: A review, Bee World 60 (1979), 59-84.

[17] M. C. Marcucci, Propolis: Chemical composition, biological properties and therapeutic activity, Apidologie 26 (1995), 83-99.

[18] N. Kalogeropoulos, S. J. Konteles, E. Troullidou, I. Mourtzinos and V. T. Karathanos, Chemical composition, antioxidant activity and antimicrobial properties of propolis extracts from Greece and Cyprus, Food Chem. 116 (2009), 452-461.

[19] M. A. Elwakil and E.-M. M. A. Hydroquinone, A promising antioxidant for managing seed-borne pathogenic fungi of peanut, Pakistan Journal of Biological Sciences 3 (2000), 374-375.

[20] J. Gómez-Estaca, A. López de Lacey, M. E. López-Caballero, M. C. Gómez-Guillén and P. Montero, Biodegradable gelatin-chitosan films incorporated with essential oils as antimicrobial agents for fish preservation, Food Microbiol. 27 (2010), 889-896.

[21] N. Chami, S. Bennis, F. Chami, A. Aboussekhra and A. Remmal, Study of anticandidal activity of carvacrol and eugenol in vitro and in vivo, Oral Microbiol. Immunol. 20 (2005), 106-111.

[22] T. Sun, D. Zhou, J. Xie and F. Mao, Preparation of chitosan oligomers and their antioxidant activity, Eur. Food Res. Technol. 225 (2007), 451-456.

[23] Y. Yang, R. Shu, J. Shao, G. Xu and X. Gu, Radical scavenging activity of chitooligosaccharide with different molecular weights, Eur. Food Res. Technol. 222 (2005), 36-40.

[24] G. G. Maghami and G. A. F. Roberts, Evaluat ion of the viscometric constants for chitosan, Die Makromolekulare Chemie. 189 (1988), 195-200.

[25] P. M. Matei, P. Martín-Ramos, M. Sánchez-Báscones, S. Hernández-Navarro, A. Correa-Guimaraes, L. M. Navas-Gracia, C. A. Rufino, M. C. Ramos-Sánchez and J. Martín-Gil, Synthesis of chitosan oligomers/propolis/silver nanoparticles composite systems and study of their activity against Diplodia seriata, Int. J. Polym. Sci. 2015 (2015), 1-11. 
[26] R. G. Woisky and A. Salatino, Analysis of propolis: Some parameters and procedures for chemical quality control, J. Apic. Res. 37 (1998), 99-105.

[27] M. Montazer, A. Shamei and F. Alimohammadi, Synthesizing and stabilizing silver nanoparticles on polyamide fabric using silver-ammonia/PVP/UVC, Prog. Org. Coat. 75 (2012), 379-385.

[28] J. L. Alcorn, The taxonomy of Helminthosporium species, Annu. Rev. Phytopathol. 26 (1988), 37-56.

[29] ISTA, International Rules for Seed Testing: Edition 2010, in, International Seed Testing Association, 2010.

[30] Y. Jiang, J. Li and W. Jiang, Effects of chitosan coating on shelf life of cold-stored litchi fruit at ambient temperature, LWT - Food Science and Technology 38 (2005) 757-761.

[31] F. C. Krzyzanowski, R. D. Vieira and J. B. França Neto, Vigor de Sementes: Conceitose Testes, Abrates, Londrina, Brasil, 1999.

[32] M. G. Canteri, R. A. Althaus, J. S. das Virgens Filho, E. A. Giglioti and C. V. Godoy, SASM-Agri: Sistema para análise e separação de médias em experimentos agrícolas pelos métodos Scott-Knott, Tukey e Duncan, Revista Bras ileira de Agrocomputação 1 (2001), 18-24.

[33] A. Kumar Tiwary, B. Sapra, G. Kaur and V. Rana, Chitosan: Modifications and Applications in Dosage Form Design, in: S. P. Davis (Ed.) Chitosan: Manufacture, Properties and Usage, Nova Science Publishers, New York, NY, USA, (2011), 71-132.

[34] P. K. Dutta, S. Tripathi, G. K. Mehrotra and J. Dutta, Perspectives for chitosan based antimicrobial films in food applications, Food Chem. 114 (2009), 1173-1182.

[35] D. F. Kendra, D. Christian and L. A. Hadwiger, Chitosan oligomers from Fusarium solani/pea interactions, chitinase/ $\beta$-glucanase digestion of sporelings and from fungal wall chitin actively inhibit fungal growth and enhance disease resistance, Physiol. Mol. Plant Pathol. 35 (1989), 215-230.

[36] H. Kuyama, Y. Nakahara, T. Nukada, Y. Ito and T. Ogawa, Stereocontrolled synthesis of chitosan dodecamer, Carbohydr. Res. 243 (1993), C1-C7.

[37] S. Hayat and A. Ahmad, Salicylic Acid: A Plant Hormone, Springer, The Netherlands, 2007.

[38] M. M. Cowan, Plant Products as Antimicrobial Agents, Clin. Microbiol. Rev. 12 (1999), 564-582.

[39] S. Shrivastava, T. Bera, A. Roy, G. Singh, P. Ramachandrarao and D. Dash, Characterization of enhanced antibacterial effects of novel silver nanoparticles, Nanotechnology 18 (2007), 225103.

[40] A. R. Silva and G. Unali, Controlled silver delivery by silver-cellulose nanocomposites prepared by a one-pot green synthesis assisted by microwaves, Nanotechnology 22 (2011), 315605. 
[41] S. Kim and N. Rajapakse, Enzymatic production and biological activities of chitosan oligosaccharides (COS): A review, Carbohydr. Polym. 62 (2005), 357-368.

[42] N. N. Duy, D. V. Phu, N. T. Anh and N. Q. Hien, Synergistic degradation to prepare oligochitosan by $\gamma$-irradiation of chitosan solution in the presence of hydrogen peroxide, Radiat. Phys. Chem. 80 (2011), 848-853.

[43] W. Xia, P. Liu, J. Zhang and J. Chen, Biological activities of chitosan and chitooligosaccharides, Food Hydrocolloids 25 (2011), 170-179.

[44] E. Savitri, S. R. Juliastuti, A. Handaratri and A. Roesyadi Sumarno, Degradation of chitosan by sonication in very-low-concentration acetic acid, Polym. Degradation Stab. 110 (2014), 344-352.

[45] P. Zou, X. Yang, J. Wang, Y. Li, H. Yu, Y. Zhang and G. Liu, Advances in characterisation and biological activities of chitosan and chitosan oligosaccharides, Food Chem. 190 (2016), 1174-1181.

[46] A. Esmaeili and A. Asgari, In vitro release and biological activities of Carum copticum essential oil (CEO) loaded chitosan nanoparticles, Int. J. Biol. Macromol. 81 (2015), 283-290.

[47] D. Altiok, E. Altiok and F. Tihminlioglu, Physical, antibacterial and antioxidant properties of chitosan films incorporated with thyme oil for potential wound healing applications, J. Mater. Sci. Mater. Med. 21 (2010), 2227-2236. 